Original Paper

\title{
Recombination Coefficient Measurement for Oxygen Recombination by Two-photon Absorption Laser Induced Fluorescence
}

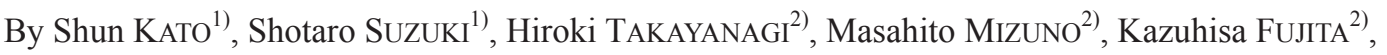 \\ Makoto MATSUI ${ }^{3)}$ and Yoshiki YAMAGIWA ${ }^{3)}$

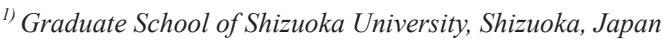 \\ 2) Japan Aerospace Exploration Agency, Tokyo, Japan \\ ${ }^{3}$ Shizuoka University, Shizuoka, Japan
}

(Received June 28th, 2013)

\begin{abstract}
Two-photon absorption laser induced fluorescence (TALIF) is applied to measure the recombination coefficient of atomic oxygen under several $\mathrm{kPa}$ pressure. The recombination coefficient of oxygen on the oxide $\mathrm{SiC}$ is estimated as $6.4 \times 10^{-3}$ at the total pressure of $2,240 \mathrm{~Pa}$, the surface temperature of $1,150 \mathrm{~K}$. From this result, we confirm that the recombination coefficient has pressure dependency.
\end{abstract}

Key Words: Surface Catalysis, Aerodynamic Heating, Re-entry, Laser Induced Fluorescence

$\begin{array}{ll}\text { Nomenclature } & \\ A & \text { Einstein coefficient } \\ A_{L} & \text { cross sectional area of laser beam } \\ b, c, B, C & \text { constant } \\ D & \text { binary diffusion coefficient } \\ E_{L} & \text { laser intensity } \\ g(v) & \text { resonance profile of frequency } \\ G & \text { photon statistic factor } \\ h & \text { Plunk's constant } \\ I_{0}(t) & \text { temporal profile of laser intensity } \\ I_{s a t} & \text { Saturation laser intensity } \\ k_{B} & \text { Boltzmann constant } \\ L & \text { boundary layer thickness } \\ M & \text { mole mass } \\ N & \text { number density } \\ Q & \text { quenching rate } \\ R & \text { gas constant } \\ R(t) & \text { excitation rate } \\ S & \text { spectrally integrated fluorescence signal } \\ T & \text { gas temperature } \\ T_{w} & \text { test piece surface temperature } \\ V^{*} & \text { average of thermal velocity } \\ \gamma & \text { recombination coefficient } \\ \Gamma(t) & \text { ionization rate } \\ \Delta^{(1)} \text { ij } & \text { collision integral } \\ v_{0} & \text { center excitation frequency } \\ v & \text { frequency } \\ v_{L} & \text { laser oscillating frequency } \\ \sigma_{i} & \text { ionization cross section } \\ \hat{\sigma}^{(2)} & \text { effective two-photon cross section } \\ \Delta \Omega & \text { collection optics solid angle } \\ \Omega_{i j}^{(1,1)} & \text { diffusion collision integral } \\ & \end{array}$

\begin{tabular}{ll}
\multicolumn{1}{c}{ Subscript } \\
1 & ground state \\
2 & excitation state \\
$i$ & under state \\
$j$ & upper state \\
$O$ & Oxygen \\
$z$ & height from test piece surface
\end{tabular}

\section{Introduction}

When a re-entry vehicle enters the atmosphere of a planet, a strong bow shock is formed in front of the vehicle's body ${ }^{1)}$. Therefore appropriate designing of a thermal protection system (TPS) for a re-entry vehicle is one of the most important issues for space missions. In such a high temperature environment, air is dissociated in the shock layer, and following recombination reactions on the TPS materials are promoted due to the surface catalysis for the case of Earth entry.

$$
\begin{gathered}
O+O \rightarrow O_{2}+5.13[\mathrm{eV}] \\
N+N \rightarrow N_{2}+9.80[\mathrm{eV}] \\
N+O \rightarrow N O+6.53[\mathrm{eV}]
\end{gathered}
$$

The schematic of this phenomenon is illustrated in Fig. 1. This characteristic is so-called the surface catalysis. The surface catalysis significantly affects the aerodynamic heating rate. It is necessary to take into account the surface catalysis on the TPS material in order to design a reliable TPS. Then, the surface catalysis is estimated by the recombination coefficient.

In our previous study, actinometry has been used to measure the recombination coefficients ${ }^{2}$. In this method, the optical emission intensity ratio of atomic oxygen to argon is used. However, it was difficult to apply under the total pressure of several $\mathrm{kPa}$ owing to the very weak optical emission. In this study, two-photon absorption laser induced fluorescence (TALIF) is applied to the atomic oxygen in order 
to evaluate the oxygen number density distribution.

TALIF is an optical diagnostics that yields the space and time resolved population in particular of atomic ground states in plasma. Schematic of the atomic oxygen by TALIF excitation transition is shown in Fig. 2.

For a precise measurement in this method, a saturation effect should be taken into account. It is a phenomenon that the ground-state number density is depleted by the high laser intensity. As a result, measurement of the number density is affected by this phenomenon. Therefore, the laser intensity should be enough low in order to avoid the saturation effect.

So far, the surface catalysis was measured using the side-arm reactor which applied TALIF by Marshall et al. in $\mathrm{NASA}^{3-5)}$. In the side-arm reactor, the total pressure is $40-60$ $\mathrm{Pa}$, and the temperature is heated upto $1,500 \mathrm{~K}$. They were measured the surface catalysis on the $\mathrm{SiC}$ in an oxide atmosphere.

In previous studies, the recombination coefficients were estimated below the total pressure of 1,000 $\mathrm{Pa}$. In the actual entry environment, the pressure is higher than the pressure of experimental environments. Therefore, this paper presents a surface catalysis measurement method for a very weak plasma emission under the total pressure of several $\mathrm{kPa}$. At first, the saturation effect is verified in a Flow-Reactor. The appropriate laser intensity without the saturation effect is estimated. Finally the surface catalysis on the $\mathrm{SiC}$ is deduced in this condition and compared with one under $10 \mathrm{~Pa}$.

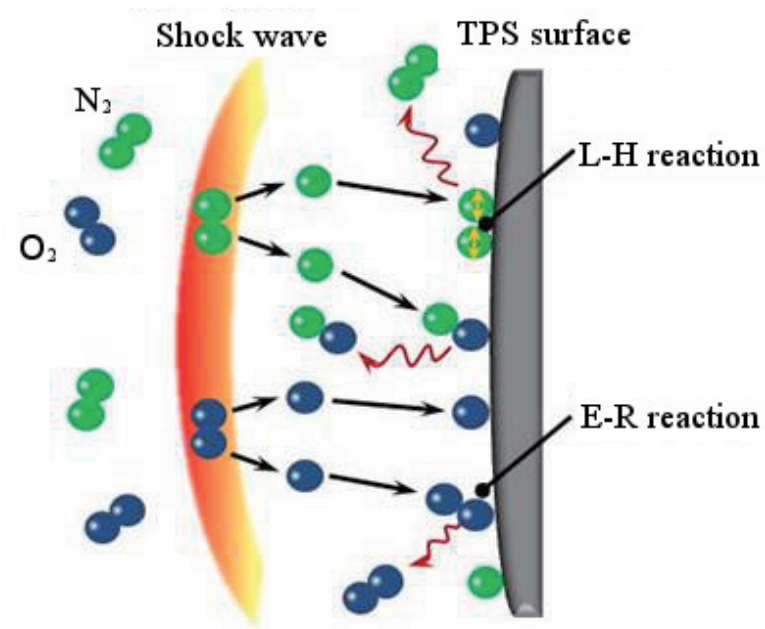

Fig. 1. Schematic of recombination model due to the surface catalysis on TPS surface.

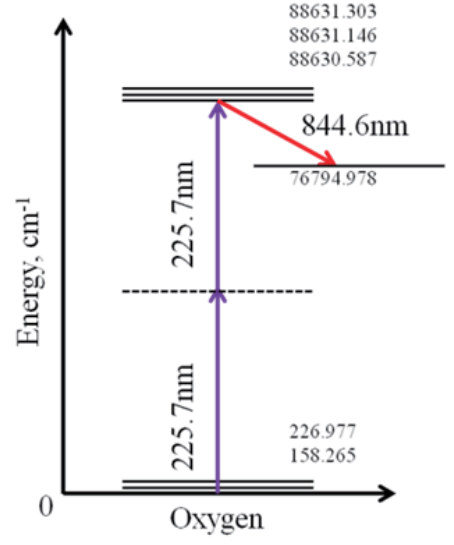

Fig. 2. Schematic of excitation transition.

2. Theory

\subsection{Two-photon absorption laser induced fluorescence}

Equations (2) and (3) show the rate equations of TALIF on the ground and excitation states ${ }^{6}$.

$$
\begin{gathered}
\frac{d}{d t} N_{2}=R(t) N_{1}(t)-(A+Q+\Gamma(t)) N_{2}(t) \\
\frac{d}{d t} N_{1}=-R(t) N_{1}(t)
\end{gathered}
$$

From these equations, the fluorescence signal spectrally integrated over the excitation line shape is proportional to the atomic number density, which is written as follows.

$$
\begin{aligned}
S \fallingdotseq & \frac{\Delta \Omega}{4 \pi} \eta_{\lambda} V \frac{A}{A+Q} \hat{\sigma}^{(2)} N_{1} G^{(2)} \\
& \cdot g\left(2 v_{L}-v_{0}\right)\left(\frac{E_{L}}{A_{L} h v_{L}}\right)^{2} \int_{0}^{\infty} I_{0}{ }^{2}(t) d t
\end{aligned}
$$

\subsection{Saturation effect}

In Eq. (4), the ground-state number density depletion is ignored. However, these factors affect the TALIF signal with high laser intensity. From Eq. (2) and (3), $d N_{2} / d t=d N_{l} / d t$ is assumed $^{7)}$. And, a photoionization is not occurred in the low laser intensity region.

$$
N_{2}=\frac{2 R(t)}{A+Q} N_{1}
$$

Here, $N_{t o t}=N_{I}+N_{2}$ is assumed.

$$
N_{2}=\frac{2 R(t)}{A+Q+2 R(t)} N_{t o t}
$$

Therefore,

$$
S_{\text {true }}=A \frac{2 R(t)}{A+Q+2 R(t)} N_{\text {tot }}
$$

Here, $R(t)$ is defined a following equation.

$$
R(t)=\hat{\sigma}^{(2)} \frac{I^{2}}{h^{2} v^{2} \tau_{L}^{2}}
$$




\subsection{Recombination coefficient}

Figure 3 shows a cylindrical coordinate in a discharge tube. A mass balance of atomic oxygen on a test piece surface is determined by a balance of particles which reached the surface by diffusion and recombined particles as shown in Eq. $(9)^{8)}$.

$$
D_{O} \frac{\partial N_{O}}{\partial z}=\left(N_{O}\right)_{z=0} \frac{V^{*}}{4} \gamma_{O}
$$

$V^{*}$ is determined by a following equation.

$$
V^{*}=\sqrt{\frac{8 R T}{\pi M_{O}}}
$$

Then, $T$ is determined by an average value with gas and test piece surface temperatures. The number density ratio is estimated by the fluorescence signal ratio. An atomic number density is inversely proportional to the gas temperature from the equation of state. A following equation shows this relation.

$$
\frac{\left(N_{O}\right)_{z=L}}{\left(N_{O}\right)_{z=0}} \propto \frac{(S)_{z=L}}{(S)_{z=0}} \frac{T_{W}}{T_{z=L}}
$$

From Eqs. (9) to (11), Eq. (12) is determined.

$$
\begin{aligned}
\gamma_{O} & =\frac{4 D_{O}}{V^{*} L} \frac{\partial\left(N_{O}\right)_{z=0}}{\partial z}=\frac{4 D_{O}}{V^{*} L}\left(\frac{\left(N_{O}\right)_{z=L}}{\left(N_{O}\right)_{z=0}}-1\right) \\
& =\frac{4 D_{O}}{V^{*} L}\left(\frac{(S)_{z=L}}{(S)_{z=0}} \frac{T_{W}}{T_{z=L}}-1\right)
\end{aligned}
$$

The binary diffusion coefficient $D_{i j}$ is defined by Gupta et $\mathrm{al}^{9)}$ as shown in Eq. (13).

$$
D_{i j}=\frac{k_{B} T}{p \Delta_{i j}^{(1)}}
$$

The collision integral $\Delta_{i j}^{(1)}$ is defined as Eq. (14).

$$
\Delta_{i j}^{(1)}=\frac{8}{3}\left(\frac{2 M_{i} M_{j}}{\pi R T\left(M_{i}+M_{j}\right)}\right)^{1 / 2} \pi \hat{\Omega}_{i j}^{(1,1)}
$$

An optimal value of the diffusion collision integral $\hat{\Omega}_{i j}^{(1,1)}$ is obtained by Wright et al ${ }^{10)}$.

Then, boundary layer thickness $L$ is determined by Eq. (15) that is fitted for fluorescence profile using the least square method.

$$
f(x)=\left\{\begin{array}{cc}
b x+c & (x \leq L) \\
\text { average } & (x>L)
\end{array}\right.
$$

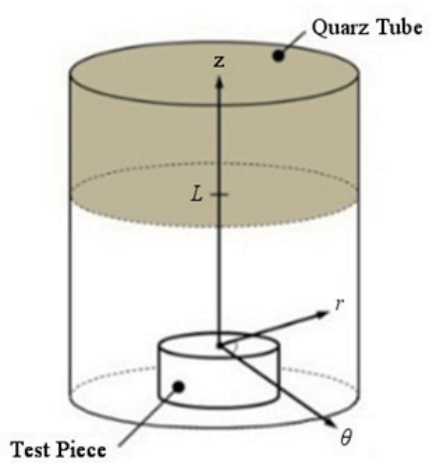

Fig. 3. Schematic of cylindrical control volume corresponding to the discharge zone.

\section{Experimental Setup}

\subsection{Measurement system}

Figure 4 shows the experimental setup to measure the recombination coefficient by TALIF.

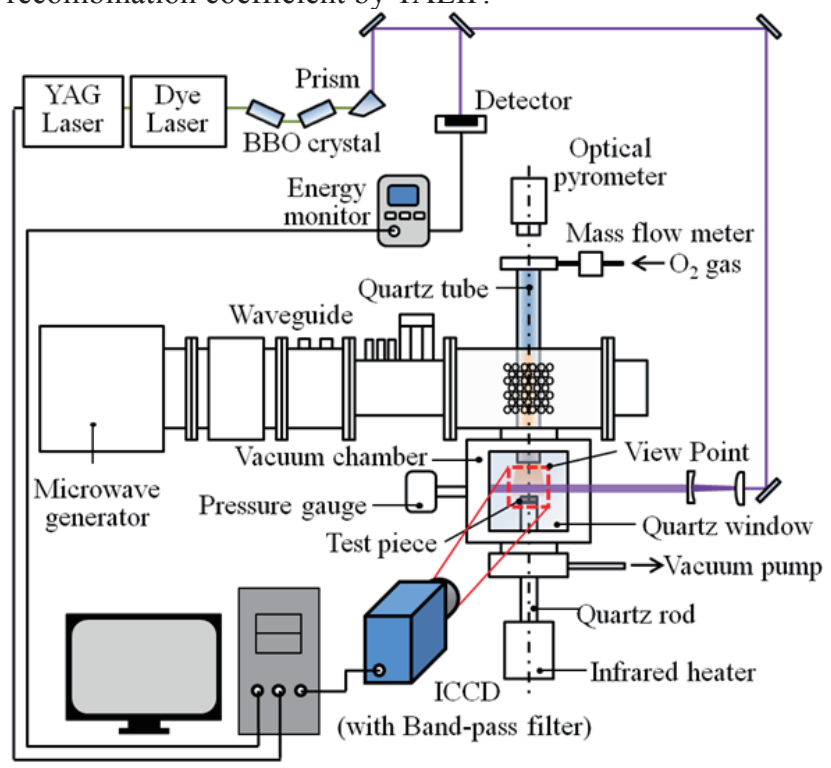

Fig. 4. Schematic of TALIF

In this paper, the recombination coefficient was derived from measurements of the fluorescence signals induced by two-photon excitation of atomic oxygen. A tunable dye laser (ScanMate 2E, Lambda Physics) pumped with a third harmonic $(355 \mathrm{~nm})$ of a Nd:YAG laser (Quanta-Ray LAB 170-10, Spectra-Physics) was used as a laser oscillator. A Coumarin 47 dissolved in a methanol was used as the dye. The target wavelength was obtained on the basis of frequency mixing in a nonlinear optical crystal. To measure the fluorescence signal, the excitation wavelength of the dye laser beam was matched to the atomic oxygen absorption transition near $225 \mathrm{~nm}$, and the fluorescence signal was recorded by an ICCD camera $(1,280 \times 1,024$ arrays with $6.7 \mathrm{~mm}$ pixels, LaVision Nanostar camera). A laser beam distance from a test piece surface was changed from $0.5 \mathrm{~mm}$ to $15 \mathrm{~mm}$ with each $0.5 \mathrm{~mm}$ to measure the spatial distribution of the TALIF signal from the atomic oxygen. In order to eliminate the emission 
from plasma, a band pass filter was inserted in front of the ICCD camera. The laser pulse and the camera exposure timing were synchronized with each other by a programmable timing unit. The laser intensity was measured by a power meter (J-50MB-LE, COHERENT).

\subsection{Flow reactor}

In this experiment, the Flow-Reactor was used as an atomic oxygen generator. This particle source generates a stationary gas flux of atomic oxygen radicals by the microwave excitation. The schematic of the Flow-Reactor is shown in Fig. 5. Oxygen gas, $10 \mathrm{sccm}$, and argon gas, $300 \mathrm{sccm}$, were admitted through the quartz tube, the inner diameter of which was $8 \mathrm{~mm}$, placed in the center of a microwave cavity which was cooled by water. Oxygen atoms were generated in the microwave discharge $(2.45 \mathrm{GHz}, 150 \mathrm{~W})$ by the electron impact dissociation. The resulting gas flux expands into the main tube, the inner diameter of which was $6.3 \mathrm{~mm}$. These connectors and tubes were made of Teflon in order to minimize wall recombination loss.

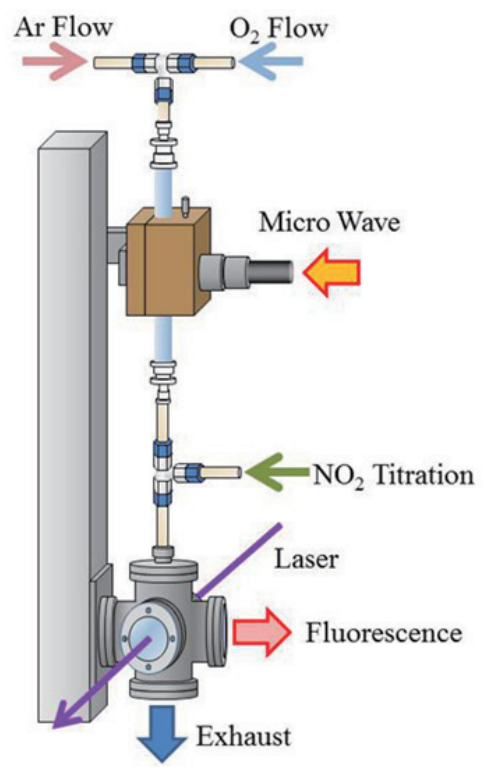

Fig. 5. Schematic of Flow Reactor.

\subsection{Microwave-discharge type surface catalysis measurement system}

The schematic of a microwave-discharged type surface catalysis measurement system is also shown in Fig.4. The 2.45 $\mathrm{GHz}$ microwave was generated from a microwave generator (Nihon Koshuha Co., Ltd, MKN-152-3S9-PS), and it formed a standing wave in a waveguide. The waveguide was cross to a discharge tube with an inner diameter of $25 \mathrm{~mm}$ and a length of $500 \mathrm{~mm}$. In the present study, the oxygen gas was supplied from the top of the discharge tube and exhausted from a bottom of a vacuum chamber by vacuum pumps. The vacuum chamber had three quartz windows, and the fluorescence from the atomic oxygen was observed through the windows. The experimental condition is shown in Table 1.
Table 1. Experimental conditions

$\begin{array}{lc}\text { Pressure, } \mathrm{kPa} & 2.2 \\ \text { Microwave power, W } & 700 \\ \text { Flow rate, sccm } & \mathrm{O}_{2}: 3,000\end{array}$

\subsection{Test piece}

In the present study, an oxide $\mathrm{SiC}$ and $\mathrm{Cu}$ were selected as test pieces. The $\mathrm{SiC}$ material was one of the most common TPS materials due to the high resistance to heat and the low catalysis. To compare the $\mathrm{SiC}$ material, the $\mathrm{Cu}$ material was also used as the test piece material with the high catalysis.

A test piece has $20 \mathrm{~mm}$ diameter and $2 \mathrm{~mm}$ thickness. Mirror-like finishing was applied to these surfaces in order to decrease the influence of surface roughness.

\section{Result and Discussion}

\subsection{Saturation effect}

The saturation effect is one of the TALIF method problems. It is caused by the ground-state depletion due to the high laser intensity. In this study, the saturation laser intensity in TALIF for atomic oxygen was verified by using the Flow-Reactor. First, the fluorescence signal was calculated using Eq. (8). The fluorescence lifetime was $85 \mathrm{~ns}$ in this Flow-Reactor. The Einstein coefficient was $3.22 \times 10^{7}$ from the NIST ${ }^{11)}$. From ref. $12)$, the effective two-photon cross section is $4.8 \times 10^{-54} \mathrm{~m}^{4} \mathrm{~s}$. The calculated result was shown in Fig. 6.

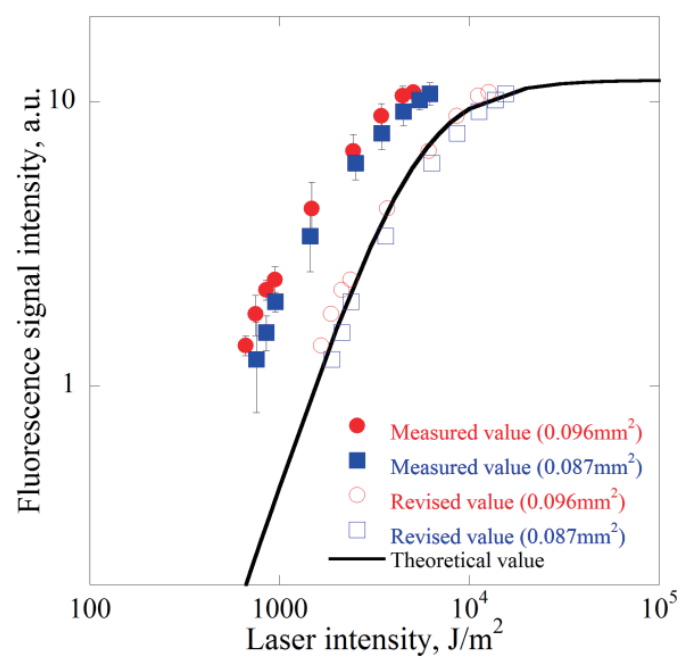

Fig. 6. Measurement result of saturation laser intensity.

The black line, closed and opened symbols showed the theoretical value from Eq. (8), measured and revised values, respectively. The error bars were estimated from the standard deviation of the measurement values. The theoretical fluorescence signal intensity was fitted to measurement saturation values by multiplying a correction factor.

Measured values showed a saturation effect. However, the values did not agree with the theoretical line. This matter might be caused from the overestimation of the spot size measured by a thermal paper, because the burn pattern might be broadened by the heat transfer. As a result, the laser intensity might be underestimated. Here, measured value was 
revised to correspond for theoretical value and plotted on Fig. 6. with square and circle hollow. Revised value was corresponded for theoretical value when the laser spot size was presumed about 2.5 times smaller than measured values. Therefore, the true laser spot size was evaluated as 0.038 and $0.035 \mathrm{~mm}^{2}$.

TALIF method has to be used in linear region of fluorescence intensity against square of laser intensity. Therefore, the laser intensity should be set lower than 1,000 $\mathrm{J} / \mathrm{m}^{2}$ in order to neglect the saturation effect.

\subsection{Recombination coefficient}

From the section 4.1., the saturation should be avoided in the test section. The laser beam power and diameter were measured in the vacuum chamber as $1 \mathrm{~mJ}$ and $3 \mathrm{~mm}$, respectively. Therefore, the laser beam intensity was estimated as $140 \mathrm{~J} / \mathrm{m}^{2}$. So, the saturation effect can be ignored in this experiment.

Figure 7 shows an example fluorescence signal image from the atomic oxygen obtained by the ICCD camera. The output picture was integrated with 10 fluorescence pictures in order to improve the signal to noise ratio. The laser beam position was moved from $0.5 \mathrm{~mm}$ to $15 \mathrm{~mm}$ height from the test piece surface.

Figure 8 shows the fluorescence signal distribution on the $\mathrm{Cu}$ surface. Over $4.5 \mathrm{~mm}$ from the surface, the fluorescence signal was constant compared to that near the surface. In this region, the atomic oxygen was not recombined by the surface catalysis. Under $4.5 \mathrm{~mm}$, the fluorescence signal was significantly decreased as the distance from the surface was decreased.

Figure 9 shows the fluorescence signal distribution on the oxide $\mathrm{SiC}$ surface at several temperatures. The decrease tendency of the fluorescence signal at the surface temperature of $1,150 \mathrm{~K}$ was slightly larger than $1,010 \mathrm{~K}$. That was, the recombination coefficient was increased as the surface temperature is increased.

The error bars show measurement error from ICCD camera and power meter. Accuracy of ICCD camera and power mater was evaluated using RMS value which is 1.8 counts and noise equivalent energy which is $8 \mu \mathrm{J}$ respectively.

The recombination coefficients were also measured by Balat et al., Cauquot et al. and Verant et al. which were shown in Fig. 10 with our results. This result shows the recombination coefficient on the $\mathrm{Cu}$ was much higher than that on oxide SiC. Recombination coefficients on the oxide $\mathrm{SiC}$ in our results showed similar tendency with other results.

In the previous study, we measured the recombination coefficients of the atomic oxygen on the $\mathrm{SiC}$ surface under 18 $\mathrm{Pa}$ using the actinometry. These values were $0.022,0.047$ and 0.142 at $930 \mathrm{~K}, 1,000 \mathrm{~K}$ and $1,150 \mathrm{~K}$ respectively. As a result, the recombination coefficient depended on the pressure, and it decreased as the pressure increased as shown in Fig. 11.

Error bar in Fig. 10 and Fig. 11 include 10\% for fitting error of Eq. (15) to fluorescence signal, 5\% for the diffusion collision integral $\hat{\Omega}_{i j}^{(1,1)(10}$ and $10 \%$ for pressure measurement. Total accuracy on the recombination coefficient is $\pm 25 \%$.

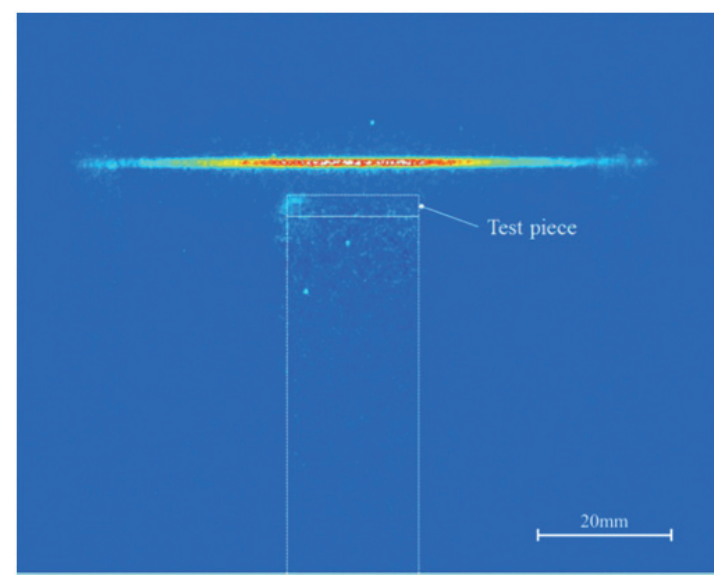

Fig. 7. Typical example of image measured at $5.5 \mathrm{~mm}$ from the test piece surface with ICCD camera

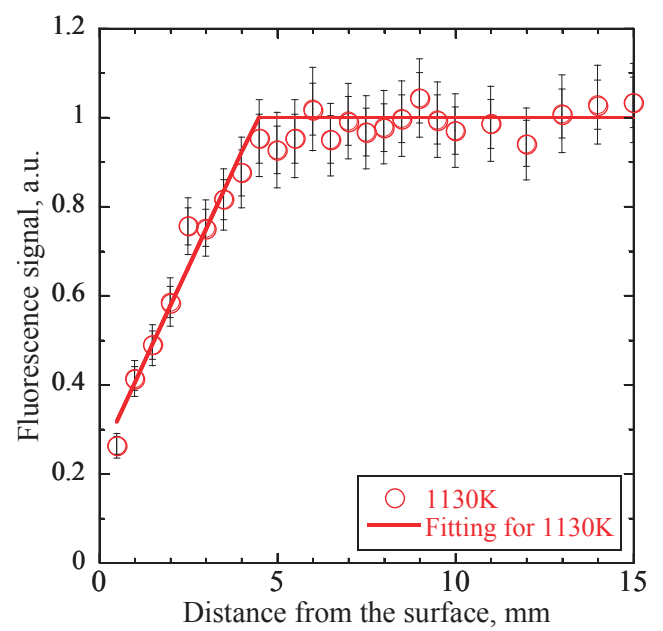

Fig. 8. Fluorescence signal intensity profile with $\mathrm{Cu}$ test piece.

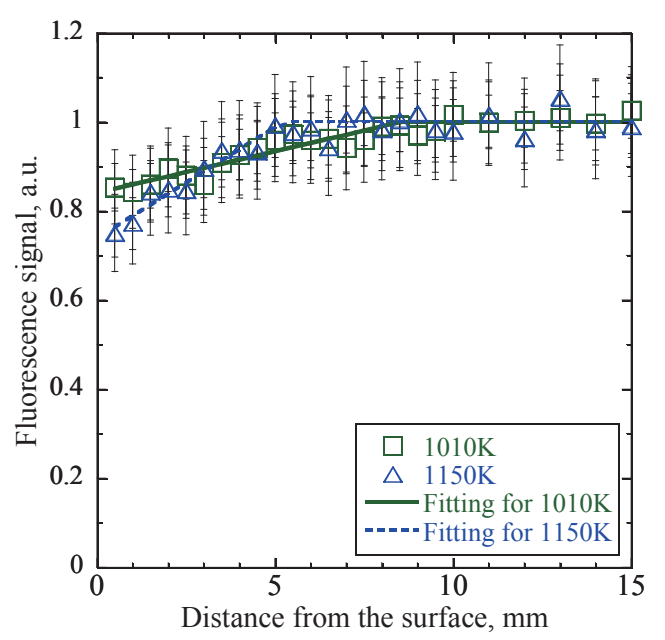

Fig. 9. Fluorescence signal intensity profiles with $\mathrm{SiC}$ test piece. 


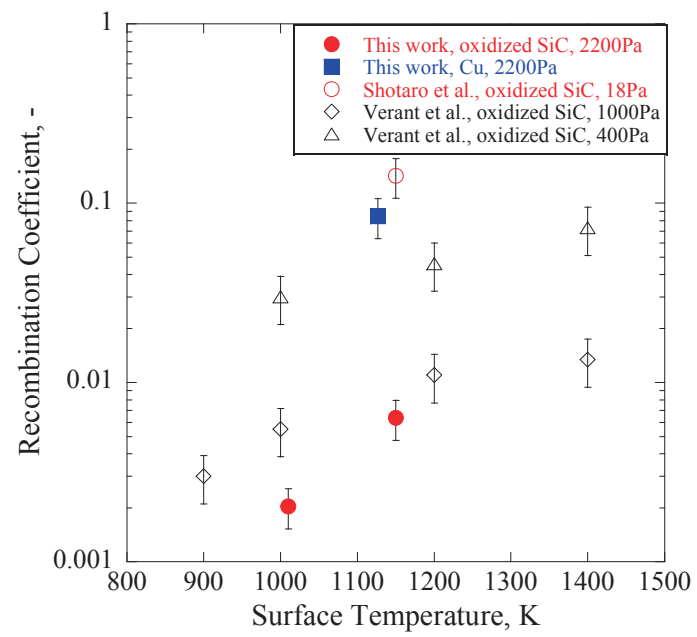

Fig. 10. Recombination coefficient of atomic oxygen recombination at $2.2 \mathrm{kPa}$ compared with the studies of Balat et al., Cauquot et al. and Verant et al. ${ }^{13-15)}$

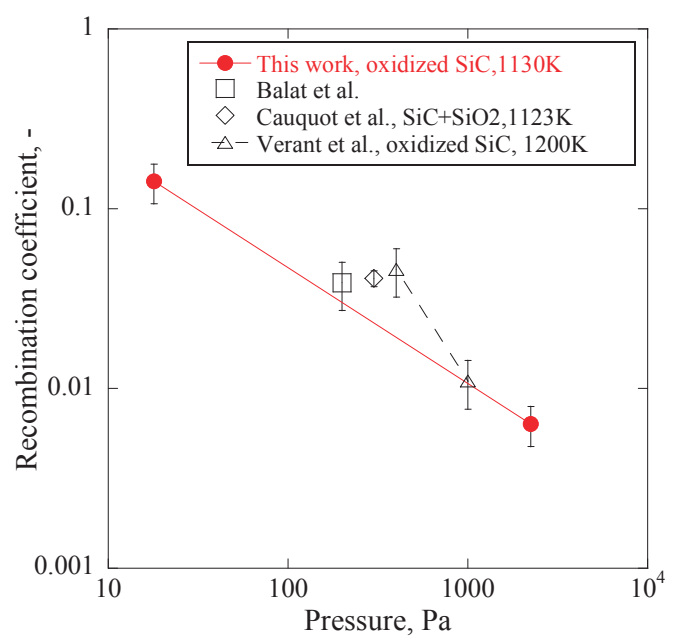

Fig. 11. Pressure dependency of recombination coefficient.

\section{Summary}

Two-photon absorption laser induced fluorescence has been applied to the atomic oxygen generated in the microwave discharged type surface catalysis measurement system developed in JAXA Chofu Space Center. The recombination coefficients under the total pressure of several $\mathrm{kPa}$ could be measured using TALIF technique. Under the total pressure of $2.2 \mathrm{kPa}$, the recombination coefficient on the oxide $\mathrm{SiC}$ was estimated as $6.36 \times 10^{-3}$ and $2.04 \times 10^{-3}$ at the surface temperature of $1,150 \mathrm{~K}$ and $1,030 \mathrm{~K}$, respectively. As a result, the pressure dependency of the recombination coefficient was expressed as an exponential function of the pressure like a eq.(16).

$$
\gamma=B \exp \left(-\frac{p}{C}\right)
$$

\section{Reference}

1) Throckmorton, D. A.: Shuttle Entry Aerothermodynamic Flight Research: The Orbiter Experiments Program, Journal of Spacecraft and Rockets, 30 (1993), pp.449-465.

2) Suzuki, S., Mizuno, M., Takayanagi, H., Fujita, K., Matsui, M., Yamagiwa, Y.: Experimental Study for Atomic Oxygen Catalytic Efficiency on TPS Surface using Microwave Discharge Plasma, AIAA paper, 2013-0742, (2013).

3) Marschal, J.: Experimental Determination of Oxygen and Nitrogen recombination Coefficient at Elevated Temperature Using Laser-induced Fluorescence, AIAA paper 1997-3879, Aug.1997.

4) Marschal, J., Chamberlain, A., Crunkleton, D., Roger, B.: Catalytic Atom Recombination on $\mathrm{ZrB}_{2} / \mathrm{SiC}$ and $\mathrm{HfB}_{2} / \mathrm{SiC}$ UltrahighTemperature Ceramic Composites, Journal of Spacecraft and Rockets, 32 (2004), pp.576-581.

5) Marschal, J., Copeland, R. A., Hwang, H.H., Wright, M. J.: Surface Catalytic Experiments on Metal Surfaces in Oxygen and Carbon Monoxide Mixture, AIAA Paper 2006-181, Jan. 2006.

6) Goehlich, A., Kawetzki, T., Dobele, H, F.: On absolute calibration with xenon of laser diagnostic methods based on two-photon absorption, Journal Of Chemical Physics, 108 (1998), pp.9362-9370.

7) Douglas, G. Fletcher.: Nonintrusive Diagnostic Strategies for Arcjet Stream Characterization, Measurement Techniques for High Enthalpy and Plasma Flows, (2000), pp.3B-1-36.

8) Balat-Pichelmin, M., Czerniak, K., Badie, J. M.: Thermal and Chemical approaches for oxy-gen catalytic recombination evaluation on ceramic materials at high temperature, Applied Sur-face Science, 120 (1997), pp.225-238.

9) Gupta, R. N., Yos, M. J., Thompson, R. A., Lee, Kam-Pui: A Review of Reaction Rates and Thermodynamic and Transport Properties for an 11-Species Air Model for Chemical and Thermal Nonequilibrium Calculations to 30000K, NASA RP-1232, 1990.

10) Wright, M. J., Bose, D., Palmer, G. E., and Levin, E.: Recommended Collision Integrals for Transport Property Computations, Part 1: Air Species, AIAA Journal, 43 (2005), pp. 2558-2564.

11) NIST Atomic Spectra Database, Version 5,2012 http://www.nist.gov/pml/data/asd.cfm.

12) Douglas, J, B., Leonard, E, J. and William, K, B.: Absolute two-photon absorption and three-photon ionization cross section for atomic oxygen, Physical Review A, 34 (1986), pp.185-198.

13) Balat-Pichelmin, M., Badie, J. M., Berjoan, R., Boubert, P.: Recombination coefficient of atomic oxygen on ceramic materials under earth re-entry conditions by optical emission spectroscopy, Chemical Physics, 291 (2003), pp.181-194.

14) Cauquot, P., Cavadias, S., Amouroux, J.: Thermal Energy Accommodation from Oxygen At-oms Recombination on Metallic Surfaces, Journal of Thermophysics and Heat Transfer, 12 (1998), pp.206-213.

15) Verant, J. L., Perron, N., Gerasimova, O., Balat-Pichelmin, M., Sakharov, V., Kolesnikov, A., Chazot, O., Omaly, P.: Microscopic and Macroscopic Analysis for TPS SiC Material under Earth and Mars Reentry Conditions, AIAA Paper 2006-7947, 2006. 